\title{
Ground state charmed meson and baryon spectra for $N_{f}=2+1+1 \mathbf{Q C D}$
}

\section{T. Rae*}

University of Wuppertal, 42119 Wuppertal, Germany

E-mail: thrae (AT) uni-wuppertal.de

\section{S. Collins}

University of Regensburg, 93040 Regensburg, Germany

E-mail: sara.collins (AT) physik.uni-regensburg.de

\section{S. Dürr}

University of Wuppertal, 42119 Wuppertal, Germany, IAS/JSC Forschungszentrum Jülich, 52425 Jülich, Germany

E-mail: durr (AT) itp.unibe.ch

\section{S. Hofmann}

University of Regensburg, 93040 Regensburg, Germany

E-mail: stefan.hofmann (AT) physik.uni-regensburg.de

We present an update on our analysis of the charmed meson ground state spectra, based on the electrically neutral subset of the BMW $N_{f}=2+1+1$ gauge configurations that use the 3-HEX smeared clover action. The analysis focuses on a systematic bootstrap evaluation of the hyperfine mass splittings of the charmonium states $J / \psi$ and $\eta_{c}$ and the singly charmed $D_{s}^{*}$ and $D_{s}$ mesons. A preliminary analysis of the charmed baryon ground state spectra is also included.

34th annual International Symposium on Lattice Field Theory

24-30 July 2016

University of Southampton, UK

${ }^{*}$ Speaker. 


\section{Introduction}

The Babar, Belle, BES III and LHCb experiments have generated a vast amount of charmed physics results; see e.g. Refs. [1,2] as a guide to the experimental literature. A key technique for understanding these results in terms of the fundamental degrees of freedom (quarks and gluons) is Lattice QCD. For reviews see e.g. Refs. [3, 4, 5, 6, 7].

This proceedings contribution provides an update to Refs. [8,9]. Our primary focus is on the hyperfine mass splittings of the charmonium states $J / \psi$ and $\eta_{c}$ and the singly charmed $D_{s}^{*}$ and $D_{s}$ mesons, with the aim to understand the often significant, cut-off effects that accompany Wilson fermion measurements. To have a good handle on this, we perform a systematic study of our lattice measurements, looping over various reasonable analysis options. We also include preliminary results for charmed baryons on one of our ensembles.

\section{Lattice setup}

The set of 27 electrically neutral 3-HEX ensembles of Ref. [10] is used for this calculation. These were generated using the clover improved Wilson action with three levels of HEX gauge link smearing [11] and $N_{f}=1+1+1+1$. Table 1 gives an overview of the ensembles used in this analysis; they are spread over four lattice spacings. These ensembles feature pion masses from $440 \mathrm{MeV}$ down to $195 \mathrm{MeV}$ and approximately physical charm and strange quark masses.

Each ensemble typically has about 400 configurations, and 64 source positions per configuration are used. One source wavefunction (a Gaussian with an approximately fixed width in physical units) is used, while at the sink two wavefunctions are used (the same Gaussian, as well as a point sink). Table 1 shows that the ensembles generally have large values of $a m_{c}$, which is a challenge for charm physics on the lattice, as it leads to large cut-off effects.

With four lattice spacings available we are in a comfortable situation in the sense that we have (assuming sufficient statistical precision) means and ways to check whether our data are in the Symanzik scaling regime. Our treatment of the statistical analysis uses 2000 bootstrap resamples, and it loops over a variety of systematic choices, which we will detail in the next section.

\section{Method and systematics}

To extract the masses (and amplitudes), we use one- and two-state fits to the correlation functions. When there are multiple channels with shared observables, we perform the fits simultane-

\begin{tabular}{cccccc}
\hline \hline$\beta$ & $a[\mathrm{fm}]$ & $a m_{c}$ & $(L / a)^{3} \times(T / a)$ & $M_{\pi}[\mathrm{MeV}]$ & Number of ensembles \\
\hline \hline 3.2 & 0.102 & 0.71 & $32^{3} \times 64$ & $235-440$ & 12 \\
3.3 & 0.089 & 0.58 & $32^{3} \times 64,48^{3} \times 64$ & $195-410$ & 6 \\
3.4 & 0.077 & 0.47 & $32^{3} \times 64,38^{3} \times 64$ & $220-405$ & 3 \\
3.5 & 0.064 & 0.35 & $32^{3} \times 64,48^{3} \times 64,64^{3} \times 96$ & $200-420$ & 6 \\
\hline \hline
\end{tabular}

Table 1: Ensemble details: lattice spacing, charm quark mass, lattice extent, range of pion masses and number of ensembles for each of the four gauge couplings. Further details are in provided in Ref. [10]. 

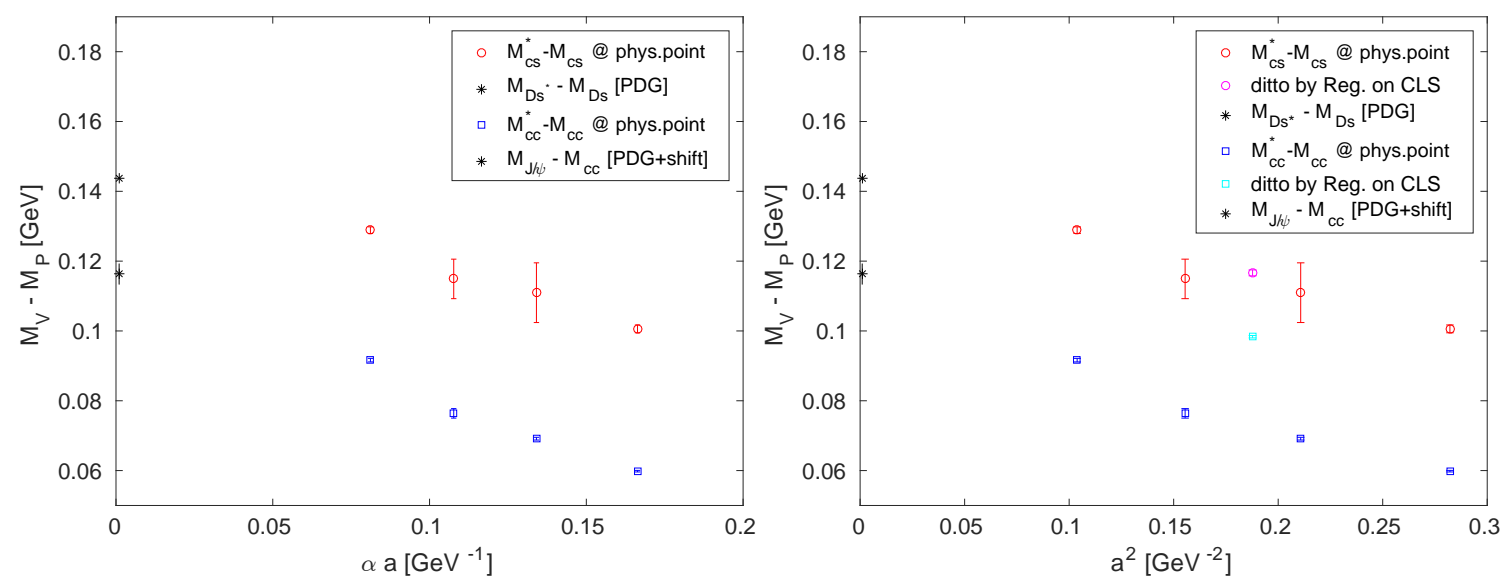

Figure 1: Results of the per- $\beta$ analyses at the physical mass point of the hyperfine splittings $M_{D_{s}^{*}}-M_{D_{s}}$ (red circles) and $M_{J / \psi}-M_{\eta_{c}}$ (blue squares) plotted versus $\alpha a$ (left) or $a^{2}$ (right), where $\alpha$ is the strong coupling constant. Since our analysis ignores disconnected contributions, the physical value of the latter mass splitting is slightly shifted (see text for details). The results of the Regensburg analysis on CLS ensembles with the lattice spacing $a \simeq 0.0855 \mathrm{fm}$ are shown in the right panel only, since non-perturbative improvement is used.

ously. To illustrate this point, for the pseudoscalar channels we simultaneously fit

$$
\begin{aligned}
\left|C_{P P}(t)\right| & =c^{2}\left(e^{-M t}+e^{-M(T-t)}\right)+\ldots \\
\left|C_{A_{4} A_{4}}(t)\right| & =d^{2}\left(e^{-M t}+e^{-M(T-t)}\right)+\ldots \\
\left|C_{P A_{4}}(t)\right|=\left|C_{A_{4} P}(t)\right| & =c d\left(e^{-M t}+e^{-M(T-t)}\right)+\ldots
\end{aligned}
$$

to the data with Gauss-Gauss wavefunctions, and we fit the same ansätze with one factor of $c$ and $d$ replaced by $c^{\prime}$ and $d^{\prime}$, respectively, to the data with the Gauss-Point smearing combination. Taking everything together, this leads to a joint fit of 8 channels with 5 shared parameters $\left(M, c^{\prime} \propto g_{P}\right.$ [the pseudoscalar coupling] and $d^{\prime} \propto f_{P}$ [the pseudoscalar decay constant] are of interest, while $c$ and $d$ do not contain any fundamental physics information).

For a careful analysis of our systematic uncertainties, we loop over various choices of the analysis strategy (on top of our bootstrap analysis which is designed to give correct statistical uncertainties for a given analysis strategy). Here we discuss the most relevant options.

(1) The selection of the fitting interval for the correlation function eqn. (3.1) uses two methods: (i) P-value maximization and (ii) $\chi^{2} /$ dof minimization (details and examples are given in [9]).

(2) Choosing $a M_{\Omega}$ to set the scale, this can be done (i) using the local ratio $M_{X} / M_{\Omega}$ ensemble by ensemble or (ii) using a $\beta$-by- $\beta$ extrapolation of $a M_{\Omega}$ to the physical mass point.

(3) There are many choices for the chiral ansätze which describe the mass splittings $f=$ $\left(M_{J / \psi}-M_{\eta_{c}}\right) / M_{\Omega}$ and $g=\left(M_{D_{s}^{*}}-M_{D_{s}}\right) / M_{\Omega}$ as a function of the quantities $x=M_{\pi}^{2} / M_{\Omega}^{2}, y=$ $\left(2 M_{K}^{2}-M_{\pi}^{2}\right) / M_{\Omega}^{2}$ and $z=M_{\eta_{c}}^{2} / M_{\Omega}^{2}$, which serve as quark mass proxies. We found two to be reasonable: (i) $f(x, y, z)=c_{0}+c_{1} x+c_{2} y+c_{3} z$ and ditto for $g(x, y, z)$ as well as (ii) $f\left(x_{\text {sea }}, y_{\text {val }}\right)=$ $c_{0}+c_{1} x_{\text {sea }}+c_{2} y_{\text {val }}$ where $x_{\text {sea }}=\left(M_{\pi}^{2}+2 M_{K}^{2}+M_{\eta_{c}}^{2}\right) / M_{\Omega}^{2}$ is used for both $f$ and $g$, while $y_{\text {val }}=$ $2 M_{\eta_{c}}^{2} / M_{\Omega}^{2}$ is used for $f\left(x_{\text {sea }}, y_{\text {val }}\right)$ and $y_{\text {val }}=\left(2 M_{K}^{2}-M_{\pi}^{2}+M_{\eta_{c}}^{2}\right) / M_{\Omega}^{2}$ is used for $g\left(x_{\text {sea }}, y_{\text {val }}\right)$. 
(4) Handling of finite-volume effects in the data; i.e. a term which is asymptotically proportional to $\exp \left(-M_{\pi} L\right)$ may be added to a given chiral ansatz or not.

(5) The continuum extrapolation may be performed with discretization terms (i) $\alpha_{s} a$ or (ii) $a^{2}$. Using this machinery, we first perform a $\beta$-by- $\beta$ analysis, i.e. we attempt an extrapolation to the physical mass point based on the 12, 6,3 or 6 ensembles that are available at the given $\beta$ (see table 1). Obviously, at $\beta=3.4$, for the time being, some of the coefficients need to be chosen with priors. The result of a particular analysis [i.e. with a specific choice for the options described under points (1) through (4) above] are shown in figure 1. We plot these hyperfine mass splittings against $\alpha a$ (left panel) and $a^{2}$ (right panel), where $\alpha=g^{2} /(4 \pi)$ is the strong coupling constant. With tree-level improvement, we know that the left panel will show a linear behavior for $a \rightarrow 0$, but in practice, this behavior was found in many instances with the second abscissa, too, over the range of accessible lattice spacings [12]. This is to say that the $\alpha a$ contribution is there, but it may be numerically subdominant to the $a^{2}$ contribution. Ideally, one would have enough lattice spacings and sufficient precision to tell these effects apart.

Fortunately, for the two hyperfine mass splittings in question, the experimental value is known, and these two quantities may thus serve as a check whether our analysis procedure yields results with a reliable estimate of the systematic uncertainty. However, there is a subtlety involved in one case. Since our data ignore disconnected contributions, we need to "correct" the experimental result of $M_{\eta_{c}}$ for this effect. Unfortunately, there is some disagreement on the size (and even the sign) of these contributions in the literature $[13,14,15,16]$. Given this situation, we decided to leave the central value unchanged, but to add a systematic uncertainty of $3 \mathrm{MeV}$ to the experimental value of $M_{J / \psi}-M_{\eta_{c}}$. This value is indicated with a black asterisk in either panel of figure 1, and also the experimental value of $M_{D_{s}^{*}}-M_{D_{s}}$ (where no "correction" is needed) is shown.

With the data in figure 1 in hand, we can say that there is a tendency to produce a sensible continuum limit, even though the splittings at the individual lattice spacings are quite far from the known continuum values (at $\beta=3.2$ we find $M_{J / \psi}-M_{\eta_{c}} \simeq 60 \mathrm{MeV}$ at the physical mass point, which is about half of the continuum value). But it is also clear that the plots raise some doubt as to whether all the data, out to $\beta=3.2$, are in the Symanzik scaling regime - perhaps we need to sacrifice the coarsest one or two lattice spacings, and perhaps we need to add an even finer lattice spacing to have a controlled continuum limit.

In such a situation, it is particularly useful to compare to another discretization which is similar in spirit but still sufficiently different to provide a check. The right panel of figure 1 includes the results from an analysis of the Regensburg group (part of the SFB-TR55 that also includes Wuppertal) on CLS ensembles for a single lattice spacing $a \simeq 0.0855 \mathrm{fm}$. Two strategies to reach the physical mass point have been implemented (fixed $2 m_{u d}+m_{s}$ versus fixed $m_{s}$ ). Since nonperturbative improvement is employed, it only makes sense to include the results in the $a^{2}$-scaling plot. While the deviations from the known continuum results are smaller than those on the 3-HEX ensembles at comparable lattice spacings, discretization effects are still sizeable and the double difference, $\left(M_{D_{s}^{*}}-M_{D_{s}}\right)-\left(M_{J / \psi}-M_{\eta_{c}}\right)$, is smaller than in the continuum.

To summarize the hyperfine splitting part, we feel it is fair to say that we have solid results in the per- $\beta$ analysis approach, which, in addition, suggest that at least the finer lattice spacings are in the Symanzik scaling regime, while for the coarser ones this is less clear. However, at the time of this writing, we lack a combined all- $\beta$ functional ansatz which yields an acceptable global fit to 

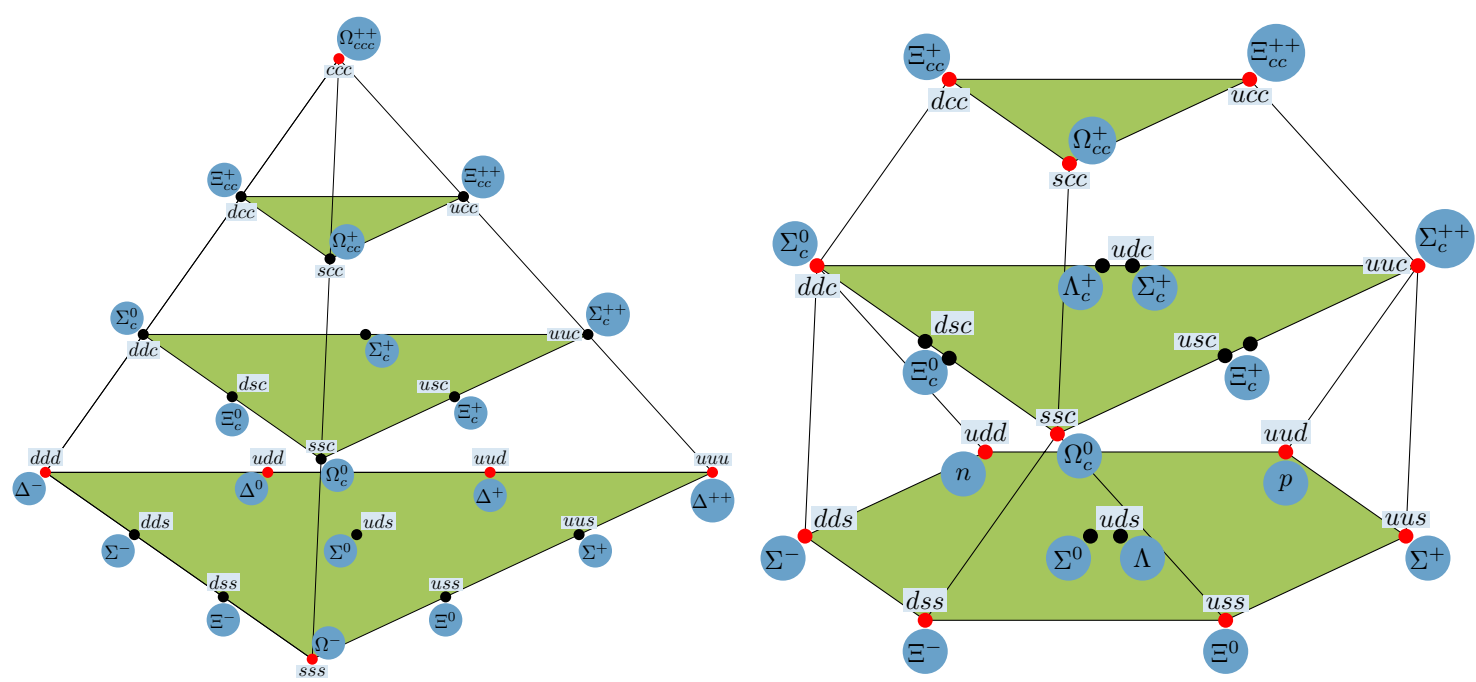

Figure 2: $\mathrm{SU}(4)$ multiplets of baryons made of $u, d, s$ and $c$ quarks (left: 20-plet, right: $20^{\prime}$-plet). The red nodes indicate those channels which are available on all of our electrically neutral 3-HEX ensembles.

all available data. We hope that more data at $\beta=3.4$ and $\beta=3.5$ will help to clarify the situation.

\section{Charmed baryon mass plateaus}

We performed a preliminary study of the signal for the charmed baryons on one of the uncharged 3-HEX ensembles. This will enable us to explore the SU(4) multiplets of baryons (20-plet and $20^{\prime}$-plet) made of $u, d, s$ and $c$ quarks (see figure 2 for an overview).

The effective mass plateaus of the totally symmetric $J^{P}=3 / 2^{+}$20-plet states $\Omega^{-}, \Omega_{c}^{0}, \Omega_{c c}^{+}$, $\Omega_{c c c}^{++}$, visualized on the front edge of the pyramid in figure 2, are shown in figure 3. Clearly the signal-to-noise ratio improves with every $s \rightarrow c$ substitution. In addition, the $\Omega_{c c c}^{++}$state assumes its plateau first. At this time, it is not clear to us whether this means that the mass gap to the first excited state is largest in this case or that the width of the Gaussian wave function (used at the source and the sink) is most appropriate in this case and a bit too narrow for the lighter states.

The effective mass plateaus of the mixed symmetry $J^{P}=1 / 2^{+} 20^{\prime}$-plet states, as visualized in the right panel of figure 2, are shown in figure 4 for zero charm content and in figure 5 for one or two valence charm quark content. Again we find an improvement of the signal-to-noise ratio with every $u, d \rightarrow s$ and every $u, d, s \rightarrow c$ replacement, but this time we do not see any marked difference in terms of how much Euclidean time is needed to assume the plateau value.

Overall it seems fair to say that baryon mass spectroscopy with charm content looks attractive in the sense that data with good signal-to-noise ratio are available. But care must be exercised to vary both $a t_{\text {ini }}$ and $a t_{\text {fin }}$ of the primary fit window within reasonable bounds, and most likely two-state fits are mandatory in order to obtain reliable baryon masses on these ensembles.

Acknowledgments: We thank Keh-Fei Liu for useful discussions regarding the disconnected mass shift of the $\eta_{c}$. We thank the Budapest-Marseille-Wuppertal collaboration for allowing us to use the configurations of Ref. [10]. This project was supported by the Deutsche Forschungsgemein- 

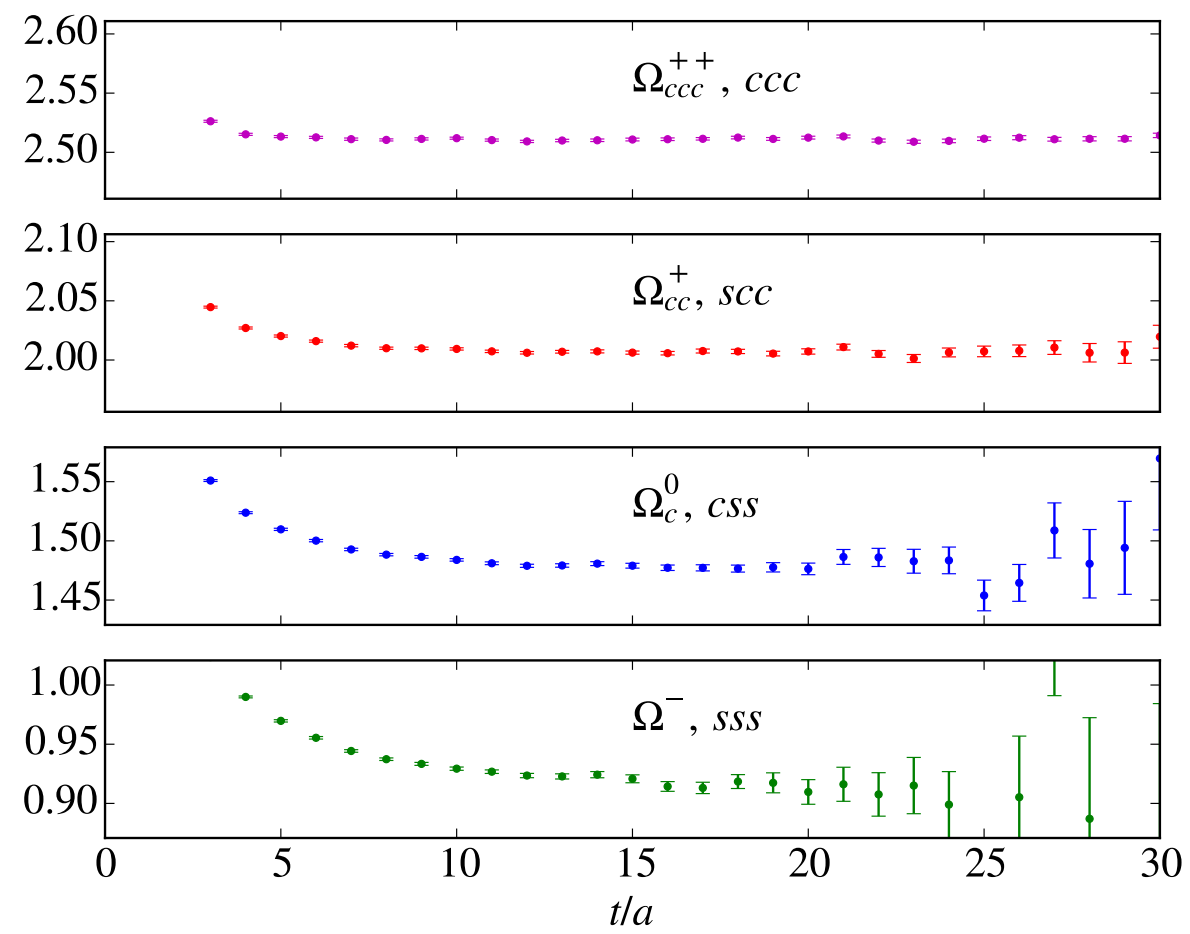

Figure 3: Effective mass plateaus of the 20-plet states $\Omega^{-}$(sss), $\Omega_{c}^{0}$ (ssc), $\Omega_{c c}^{+}$(scc), $\Omega_{c c c}^{++}$(ccc) on one of our 3-HEX ensembles. The $y$-ranges of the four panels have the same width of 0.15 in lattice units.

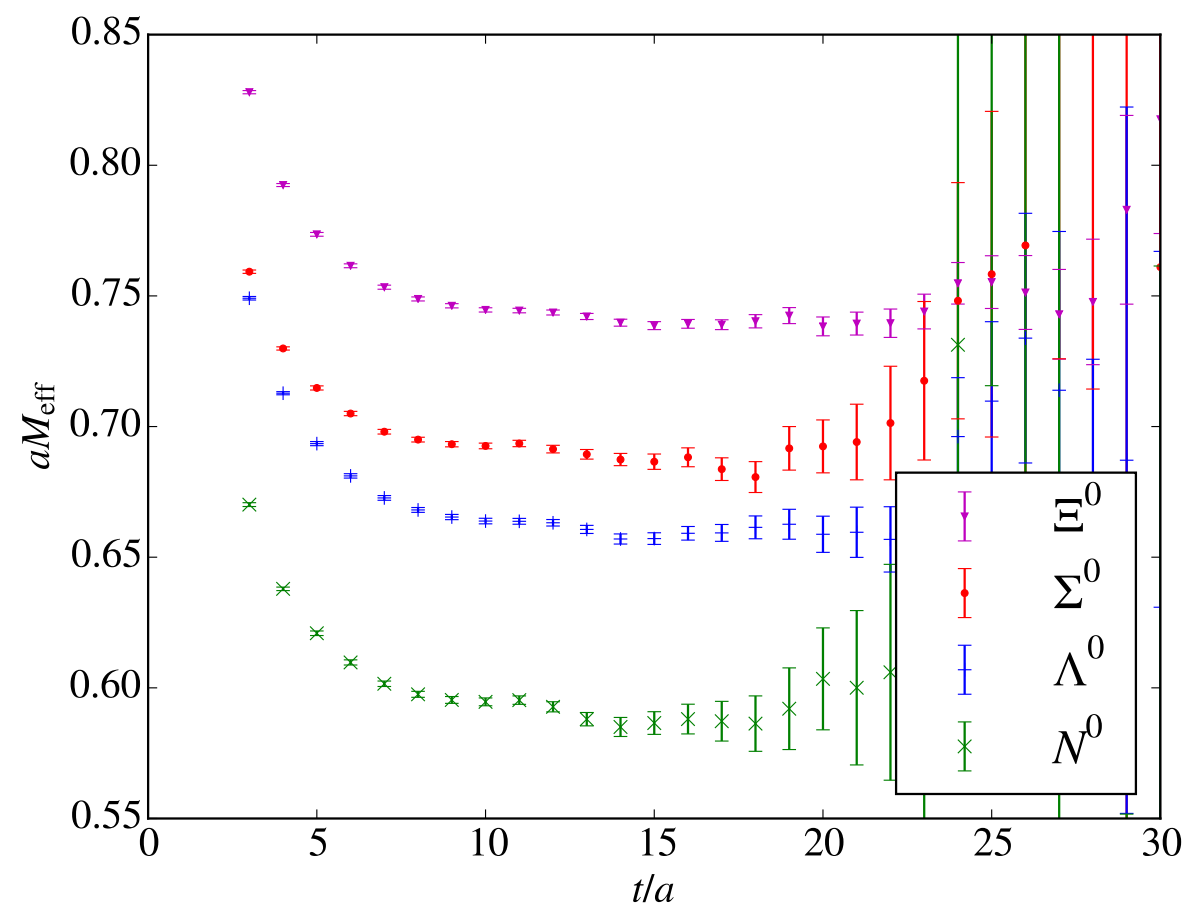

Figure 4: Effective mass plateaus of the $c=0$ baryon octet states (ground floor of the 20'-plet in figure 2). 

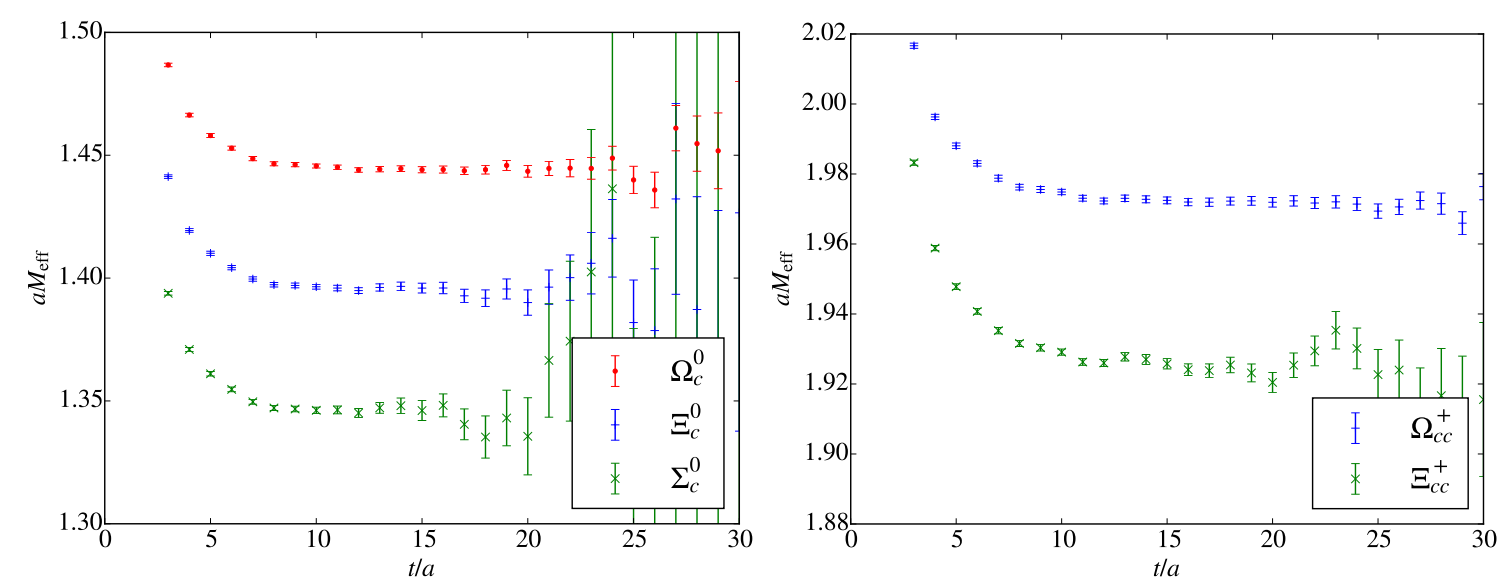

Figure 5: Effective mass plateaus of some of the singly and doubly charmed $20^{\prime}$-plet states (1st and 2nd floor in the right panel of figure 2 on the same ensemble as in figures 3 and 4.

schaft grant SFB/TRR-55. Computations were performed on JUQUEEN and JUROPA at IAS/JSC Forschungszentrum Jülich GmbH, on Turing at IDRIS in Orsay, on SuperMUC at Leibniz Supercomputing Centre in München and on Hermit at the High Performance Computing Center in Stuttgart.

\section{References}

[1] N. Brambilla et al., Eur. Phys. J. C 71 (2011) 1534 [arXiv:1010.5827 [hep-ph]].

[2] Y. Amhis et al. [Heavy Flavor Averaging Group (HFAG) Collaboration], arXiv:1412.7515 [hep-ex].

[3] A. X. El-Khadra, PoS LATTICE 2013 (2014) 001 [arXiv:1403.5252 [hep-lat]].

[4] C. M. Bouchard, PoS LATTICE 2014 (2015) 002 [arXiv:1501.03204 [hep-lat]].

[5] C. Pena, PoS LATTICE 2015 (2016) 013 [arXiv:1607.01173 [hep-lat]].

[6] R. Zhou, PoS LATTICE 2016 (2017) 012.

[7] S. Aoki et al., arXiv:1607.00299 [hep-lat].

[8] G. Bali et al., PoS LATTICE 2011 (2011) 135 [arXiv:1108.6147 [hep-lat]].

[9] T. D. Rae and S. Dürr, PoS LATTICE 2015 (2016) 094 [arXiv:1509.02381 [hep-lat]].

[10] S. Borsanyi et al., Science 347 (2015) 1452 [arXiv:1406.4088 [hep-lat]].

[11] S. Capitani, S. Dürr and C. Hoelbling, JHEP 0611 (2006) 028 [hep-lat/0607006].

[12] S. Dürr et al., JHEP 1108 (2011) 148 [arXiv:1011.2711 [hep-lat]].

[13] T. Feldmann, P. Kroll and B. Stech, Phys. Lett. B 449 (1999) 339 [hep-ph/9812269].

[14] E. Follana et al. [HPQCD and UKQCD Collaborations], Phys. Rev. D 75 (2007) 054502 [hep-lat/0610092].

[15] H. Y. Cheng, H. n. Li and K. F. Liu, Phys. Rev. D 79 (2009) 014024 [arXiv:0811.2577 [hep-ph]].

[16] L. Levkova and C. DeTar, Phys. Rev. D 83 (2011) 074504 [arXiv:1012.1837 [hep-lat]]. 